\title{
CHARACTERIZING WHEN $R[X]$ IS INTEGRALLY CLOSED
}

\author{
THOMAS G. LUCAS *
}

(Communicated by Louis J. Ratliff, Jr.)

\begin{abstract}
Unlike the situation when dealing with integral domains, it is not always the case that the polynomial ring $R[X]$ is integrally closed when $R$ is an integrally closed commutative ring with nonzero zero divisors. In the main theorem it is shown that for an integrally closed reduced ring $R, R[X]$ is not integrally closed if and only if there exists a finitely generated dense ideal $J$ and an $R$-module homomorphism $s \in \operatorname{Hom}_{R}(J, R)$ such that $s$ is integral over $R$ and $s$ is not defined by multiplication by a fixed element of $R$. As a corollary it is shown that $R[X]$ is integrally closed if and only if $R$ is integrally closed in $T(R[X])$, the total quotient ring of $R[X]$.
\end{abstract}

\section{INTRODUCTION}

In what follows all of the rings are assumed to be commutative with nonzero identity. When we say that a ring $R$ is integrally closed we mean that $R$ is integrally closed in $T(R)$, the total quotient ring of $R$. An element of $R$ which is not a zero divisor is said to be regular and an ideal $I$ of $R$ is said to be dense if $r I=(0)$ implies $r=0$. If the only finitely generated dense ideals are those which contain regular elements, $R$ is said to have property $A$. Finally, the set of minimal prime ideals of $R$ is denoted by $\operatorname{Min} R$.

An exercise in Gilmer's book [G, Exercise 11, p. 100] essentially asks the reader to determine necessary and sufficient conditions in order that the polynomial ring $R[X]$ be integrally closed. The solution is not that $R$ is integrally closed for unlike the situation when dealing with an integral domain, it is not the case that $R[X]$ is integrally closed when $R$ is integrally closed. Of course if $R$ contains a nonzero nilpotent element $k$, then $R[X]$ is not integrally closed since $k / X$ is not a polynomial but is integral over $R[X]$. But even if $R$ is an integrally closed reduced ring, $R[X]$ need not be integrally closed. We present

Received by the editors March 25, 1988 and, in revised form, June 6, 1988. This paper was presented during the $841 \mathrm{st}$ meeting of the American Mathematical Society in Knoxville, TN on March 26, 1988.

1980 Mathematics Subject Classification (1985 Revision). Primary 13B20; Secondary 13F20, $13 \mathrm{~B} 25$.

Key words and phrases. Reduced ring, integrally closed, complete ring of quotients, dense ideal.

Supported in part by funds from the Foundation of the University of North Carolina at Charlotte and the State of North Carolina. 
one such ring in our Example 3 and others can be found in various places including [BCM, Example 1], [ $\mathrm{A}_{1}$, p. 69], and [ $\mathrm{Lu}_{2}$, Example 1.4].

Various authors have produced sufficient conditions for $R[X]$ to be integrally closed. In the mid-1970s, Gilmer proved, but did not publish, the following: If $R$ is an integrally closed ring such that $R_{M}$ is an integrally closed domain for every maximal ideal $M$, then $R[X]$ is integrally closed. Later, in a 1980 paper $\left[\mathrm{A}_{1}\right.$ ], Akiba gave an independent proof of the above result and used it to prove his Theorem 2.1: Let $R$ be an integrally closed reduced ring for which $\operatorname{Min} R$ is compact (in the Zariski topology). Then $R[X]$ is integrally closed if and only if $T(R)$ is von Neumann regular. In Proposition 9 of [Q], Quentel proved that for a reduced ring $R, T(R)$ is von Neumann regular if and only if $\operatorname{Min} R$ is compact and $R$ has property $A$. With this we can restate Akiba's result as: Let $R$ be an integrally closed reduced ring for which $\operatorname{Min} R$ is compact. Then $R[X]$ is integrally closed if and only if $R$ has property $A$. Without the assumption that $\operatorname{Min} R$ is compact, Akiba proved $R$ having property $A$ is sufficient for $R[X]$ to be integrally closed when $R$ is reduced and integrally closed. Using this result together with two others of Akiba's, namely [ $A_{1}$, Corollary 1.2] and [ $\mathrm{A}_{2}$, Lemma 1.1], it is possible to prove the following: If $R$ is an integrally closed reduced ring for which $R_{M}$ has property $A$ and is integrally closed for each maximal ideal $M$, then $R[X]$ is integrally closed. A proof of the above result can be found in $[H, p .103]$. Whether this result says anything new is not known. But the ring in our Example 2 shows that $R$ need not be locally integrally closed in order for $R[X]$ to be integrally closed. This same ring, as well the ring in $\left[\mathrm{A}_{1}\right.$, Example], shows that $R$ need not have property $A$ in order for $R[X]$ to be integrally closed.

The most recently discovered sufficient condition of which we are aware involves rings which are strongly Prüfer. A ring $R$ is said to be strongly Prüfer if every finitely generated dense ideal is locally principal. In [D], Dixon proved the following: If $R$ is an integrally closed reduced ring and $T(R)$ is strongly Prüfer, then $R[X]$ is integrally closed. A proof of this result can also be found in $[\mathrm{H}, \mathrm{p} .118]$ as well as an example to show that $T(R)$ need not be strongly Prüfer in order for $R[X]$ to be integrally closed [H, Example 18]. The ring of Example 17 in $[\mathrm{H}]$ shows that a strongly Prüfer reduced ring need not have property $A$.

The question now is what do these various sufficient conditions have in common. The answer (in some sense) lies in considering the examples mentioned above where $R$ is an integrally closed reduced ring and $R[X]$ is not integrally closed. In each case there is a quotient of polynomials $f / g \in T(R[X]) \backslash R[X]$ such that not only is $f / g$ integral over $R[X]$ but integral over $R$ as well. Moreover, if $f_{j}$ and $g_{j}$ denote the $j$ th coefficient of $f$ and $g$, respectively, then $(f / g) g_{j}=f_{j}$ so that $f / g$ defines an $R$-module homomorphism from the ideal $c(g)=\left(g_{0}, g_{1}, \ldots, g_{n}\right)$ of $R$ to $R$. As part of our main theorem (Theorem 4 ) we show that existence of such a quotient is both necessary 
and sufficient for $R[X]$ to fail to be integrally closed. Hence, we have that $R[X]$ is integrally closed if and only if $R$ is integrally closed in $T(R[X])$ (Corollary 5).

Any undefined notation or terminology is standard as in [AM] or [G]. Also the results due to Akiba, Dixon, Gilmer, and Quentel can all be found in [H].

\section{WhEN $R[X]$ IS INTEGRALLY CLOSED}

As noted earlier, if $R$ contains a nonzero nilpotent element, then $R[X]$ is never integrally closed. Hence, except for Corollary 5 , all of our results are stated for reduced rings.

To motivate our characterization of when $R[X]$ is integrally closed we start with two examples. The rings presented in these two examples are so-called $A+B$ rings. Before presenting our examples, we describe the basic method of construction of $A+B$ rings and list some of their properties in Lemma 1.

Let $D$ be a domain and let $\mathscr{P}$ be a set of prime ideals of $D$ such that $\bigcap_{P_{u} \in \mathscr{P}} P_{\alpha}=(0)$ and $\bigcup_{p_{n} \in \mathscr{P}} P_{\alpha}$ equals the set of nonunits of $D$. Let $I=\mathscr{A} \times N$, where $\mathscr{A}$ is an index set for $\mathscr{P}$ and $N$ is the set of natural numbers. For each $i=(\alpha, n) \in I$, let $D_{i}=D / P_{\alpha}$ and $K_{i}=q f\left(D_{i}\right)$. Let $A$ be the canonical image of $D$ in $\prod_{i \in I} D_{i}$ and let $R=A+B$ where $B=\sum_{i \in I} K_{i}$.

Lemma 1. Let $R=A+B$ be a ring formed in the above manner. Then

(a) For each $r \in R, r$ can be written uniquely as $r=a+b$ where $a \in A$ and $b \in B$.

(b) For $r \in R, r$ is a zero divisor if and only if for some $i \in I$, the $i$ th component $(r)_{i}$ of $r$ equals zero.

(c) If $r \in R$ is not a zero divisor, it is a unit. Hence, $R=T(R)$.

(d) $A$ is canonically isomorphic to $D$.

(e) $R$ has property $A$ if and only if for every finitely generated proper ideal $J$ of $D, J \subset P_{\alpha}$ for some $P_{\alpha} \in \mathscr{P}$.

(f) If $\cap D_{P_{n}}=D$, then $R[X]$ is integrally closed.

Proof. As we need the lemma only for our examples we will only sketch the proofs. For a more detailed account of $A+B$ rings see [H, §26], [ $\left.\mathrm{Lu}_{1}\right]$ and $\left[\mathrm{Lu}_{2}\right]$.

The proofs of (a)-(d) are straightforward, that of (c) following from the fact that $B$ is a direct sum of fields and the assumption that $\cup P_{\alpha}$ contains all of the nonunits of $D$. The proof of (d) follows from the assumption that $\cap P_{\alpha}=(0)$.

The proof of (e) follows from showing that for an ideal $H$ of $R, H$ has a nonzero annihilator if and only if for some $i \in I,(r)_{i}=0$ for all $r \in H$.

The proof of ( $f$ ) is more involved than the others and a more detailed proof can be found in $\left[\mathrm{Lu}_{2}\right]$. Essentially the proof has two parts. The first is to show that if $b \in T(R[X])$ is integral over $R[X]$, then $b$ can be written as $b=f / g+k$, where $k \in B[X]$ and $f, g \in D[X]$ with $f / g$ integral over $D[X]$. Hence, using the proof of (e), we have that the content of $g$ (as a polynomial 
over $D$ ) is not contained in any $P_{\alpha}$ since $g$ is not a zero divisor of $R[X]$. Thus $g$ has unit content as a polynomial over $D_{P_{n}}$. As $f / g$ is integral over $D[X]$, it is also integral over $D_{P_{n}}[X]$. By the content formula we get that $f / g$ reduces to a polynomial over $D_{P_{n}}$. Whence, if $\cap D_{P_{n}}=D, f / g \in D[X]$ and $R[X]$ is integrally closed.

Example 2. Let $K$ be a field and let $D=K\left[Z^{2}, Z^{3}, Y\right]_{M}$ with $M=$ $\left(Z^{2}, Z^{3}, Y\right)$. Let $\mathscr{P}$ be the set of height one primes of $D$ and form the corresponding $A+B$ ring $R$. Viewing $M D$ as an ideal of $A$, we see that $N=M D+B$ is a maximal ideal of $R$ and that $R_{N}$ is isomorphic to $D$. Hence $R$ is not locally an integrally closed domain. Moreover, $R$ does not have property $A$ since $M D$ is not contained in any height one prime of $D$. It is elementary to show that $(D: M D)=D$ so that $M D$ is not a maximal prime of a principal ideal. Thus by [K, Theorem 53], $D=\cap D_{P_{s t}}$ and $R[X]$ is integrally closed.

We use a similar domain in our next example but add $Z Y$ to the definition of $D$ so that now the maximal ideal $M D=\left(Z^{2}, Z^{3}, Z Y, Y\right) D$ is divisorial, that is $(D:(D: M D))=M D$.

Example 3. Let $K$ be a field and let $D=K\left[Z^{2}, Z^{3}, Z Y, Y\right]_{M}$ with $M=$ $\left(Z^{2}, Z^{3}, Z Y, Y\right)$. As before let $\mathscr{P}$ be the set of height one primes of $D$ and form the corresponding $A+B$ ring $R$. We shall show that $R[X]$ is not integrally closed.

For ease of notation we begin by setting $f_{0}=Z Y, f_{1}=Z^{2} Y, f_{2}=Z^{3}$, $f_{3}=Z^{4}$, and $g_{0}=Y, g_{1}=Z Y, g_{2}=Z^{2}$, and $g_{3}=Z^{3}$. Define polynomials $f, g \in R[X]$ by $f(X)=f_{3} X^{3}+f_{2} X^{2}+f_{1} X+f_{0}$ and $g(X)=g_{3} X^{3}+g_{2} X^{2}+$ $g_{1} X+g_{0}$. Then $g$ is not a zero divisor of $R[X]$ since the content of $g$ in $D$ is $M D$. As $Z \notin D, f / g \notin R$. But $(f / g)^{2}=Z^{2}$ so that $f / g$ is integral over $R$. Hence, $R[X]$ is not integrally closed.

Remark. Observe that for the polynomials $f$ and $g$ of Example 3, not only is $f / g$ integral over $R$ but $(f / g) g_{i}=f_{i}$ for each $i$. Hence, multiplication by $f / g$ defines an $R$-module homomorphism from the dense ideal $\left(g_{0}, g_{1}, g_{2}, g_{3}\right)$ to $R$ and this homomorphism is integral over $R$. In Theorem 4 we show that such a situation arises whenever $R[X]$ is not integrally closed.

Before presenting the theorem we recall a few facts from Lambek's book concerning $Q(R)$, the complete ring of quotients of $R$ [La, pp. 36-46].

We begin with the definition.

Let $J_{1}$ and $J_{2}$ be dense ideals of $R$ and let $f_{i} \in \operatorname{Hom}_{R}\left(J_{i}, R\right)$ for $i=1,2$. As $J_{1} \cap J_{2}$ is also dense, we may define $f_{1}+f_{2}$ as an $R$-module homomorphism from $J_{1} \cap J_{2}$ to $R$. To define the product $f_{1} f_{2}$ note that $f_{1} f_{2} \in$ $\operatorname{Hom}_{R}\left(f_{2}^{-1} J_{1}, R\right)$. To make $Q(R)$ into a commutative ring, define an equivalence relation $\Theta$ on the homomorphisms above by $f_{1} \Theta f_{2}$ if and only if 
$f_{1}(u)=f_{2}(u)$ for each $u \in J_{1} \cap J_{2}$. With this definition it turns out that not only is $Q(R)$ a commutative ring but it is also von Neumann regular provided $R$ is reduced.

For $a / b \in T(R)$, we may define a homomorphism $f \in \operatorname{Hom}_{R}(b R, R)$ by $f(b r)=a r$. Hence, we have that both $R$ and $T(R)$ embed naturally in $Q(R)$. Moreover, $T(R[X])$ embeds in $T(Q(R)[X])$ since any finitely generated ideal $J$ of $R$ has a nonzero annihilator in $R$ if and only if $J$ has a nonzero annihilator in $Q(R)$.

If $R$ is reduced, then $Q(R)[X]$ is integrally closed since $Q(R)$. is von Neumann regular (see for example [GP, p. 224]). Hence, if we let $S$ be the integral closure of $R$ in $Q(R)$, we have that $S[X]$ is the integral closure of $R[X]$ in $T(Q(R)[X])$.

Theorem 4. Let $R$ be an integrally closed reduced ring and let $S$ be the integral closure of $R$ in $Q(R)=Q$. Then the following are equivalent.

(1) $R[X]$ is not integrally closed.

(2) There exists an element $s \in S \backslash R$ such that $s=f / g \in T(R[X])$.

(3) There exists an element $f / g \in T(R[X]) \backslash R[X]$ which is integral over $R$

(4) There exists a finitely generated dense ideal $J$ of $R$ and an $R$-module homomorphism $s$ from $J$ to $R$ such that $s \in S \backslash R$.

Proof. Obviously, (3) implies (1). The equivalence of (2) and (3) follows from the remarks preceding the theorem.

To see that (4) implies (1), (2), and (3), let $J=\left(a_{0}, \ldots, a_{n}\right)$ be a dense ideal of $R$ and let $s \in \operatorname{Hom}_{R}(J, R) \backslash R$ be integral over $R$.

For each $j=0,1, \ldots, n$ let $b_{j}=s\left(a_{j}\right)=s a_{j}$ and set $f(X)=b_{n} X^{n}+\cdots+b_{0}$ and $g(X)=a_{n} X^{n}+\cdots+a_{0}$. Then as an element of $T(Q[X]), f / g=s$. As $s$ is not a polynomial over $R, f / g \in T(R[X]) \backslash R[X]$ and $f / g$ is not only integral over $R[X]$ but over $R$ as well.

It remains to show that (1) implies (4). To this end assume that $R[X]$ is not integrally closed and let $f / g \in T(R[X]) \backslash R[X]$ be integral over $R[X]$. Viewed as an element of $T(Q[X])$ we may write $f / g$ as $f / g=s(X)=s_{k} X^{k}+\cdots+s_{0} \in$ $S[X]$ with some $s_{i} \in S \backslash R$. Since $s(X)$ is a polynomial we may pick $f$ and $g$ so that the degree of $s(X)$ is minimal. Our intent is to show that $k=0$ and in the process that $f / g=s_{0}$ defines an $R$-module homomorphism from the content of $g$ into $R$.

Claim 1. $s_{k}, s_{0} \in S \backslash R$.

If $s_{k} \in R$, then $f / g-s_{k} X^{k}=s_{k-1} X^{k-1}+\cdots+s_{0}$ is both an element of $T(R[X])$ and integral over $R[X]$. Likewise, if $s_{0} \in R$, then $\left(f / g-s_{0}\right) X^{-1}=$ $s_{k} X^{k-1}+\cdots+s_{1}$ is both an element of $T(R[X])$ and integral over $R[X]$. As the degree of $s(X)$ was assumed to be minimal neither $s_{k}$ nor $s_{0}$ can be in $R$. 
Write $g(X)=g_{m} X^{m}+\cdots+g_{0}$. We will show that $s_{k} g_{j} \in R$ for each $j$. With this we may conclude that $k=0$ as otherwise we would have

$$
\frac{f}{g}-\frac{g s_{k} X^{k}}{g}=s_{k-1} X^{k-1}+\cdots+s_{0} \in T(R[X]) \backslash R[X] .
$$

Claim 2. For each $i$ and $j, s_{i} g_{j} \in R$.

Obviously, $s_{k} g_{m} \in R$. Hence, by multiplying both sides of $f / g=s(X)$ by $g_{m}$ and rearranging we get that $g_{m} f / g-g_{m} s_{k} X^{k}=g_{m} s_{k-1} X^{k-1}+\cdots+g_{m} s_{0}$. As the degree of $s(X)$ was minimal, $g_{m} s_{i} \in R$ for each $i$. Proceeding inductively we get first that $g_{m-1} s_{k} \in R$ as both $g_{m} s_{k-1}$ and $g_{m} s_{k-1}+g_{m-1} s_{k}$ are in $R$. Hence, as above $g_{m-1} s_{i} \in R$ for each $i$. Continuing this process we get $s_{i} g_{j} \in R$ for each $i$ and $j$. In particular, $s_{k} g_{j} \in R$ for each $j$.

As noted above, with $s_{k} g_{j} \in R$ for each $j$ we have that $k=0$ and so $f / g=$ $s_{0} \in S$. Moreover, $(f / g) g_{j}=f_{j}$ for each $j$ since $f=s_{0} g$. As the content of $g$ is a finitely generated dense ideal of $R$, we have that multiplication by $f / g$ defines an $R$-module homomorphism from the content of $g$ to $R$.

Our first two corollaries restate the above result in the positive.

Corollary 5. Let $R$ be a ring. Then $R[X]$ is integrally closed if and only if $R$ is integrally closed in $T(R[X])$.

Proof. For $R$ an integrally closed reduced ring, the statement is essentially the contrapositive of Theorem 4 . In the event that $R$ is either not reduced or not integrally closed, the statement holds since neither $R$ nor $R[X]$ can be integrally closed in $T(R[X])$.

Corollary 6. Let $R$ be a reduced ring. Then $R[X]$ is integrally closed if and only if for every finitely generated dense ideal $J$ of $R,\left(R:{ }_{Q}\right) \cap S=R$.

We conclude by giving a new and much condensed proof of Akiba's Theorem 3.2 in $\left[\mathrm{A}_{1}\right]$.

Corollary 7. Let $R$ be an integrally closed reduced ring with property $A$. Then $R[X]$ is integrally closed.

Proof. It is routine to verify that $R$ has property $A$ if and only if $T(R)$ has property $A$. Hence, as $R[X]$ is integrally closed in $T(R)[X]$ we may assume that $R=T(R)$.

In a total quotient ring with property $A$ the only finitely generated dense ideal is the ring itself. Hence, $R[X]$ is integrally closed since $\operatorname{Hom}_{R}(R, R)=R$.

\section{REFERENCES}

[ $A_{1}$ ] T. Akiba, Integrally-closedness of polynomial rings, Japan J. Math. 6 (1980), 67-75.

[ $A_{2}$ ] T. Akiba, On the normality of $R(X)$, J. Math. Kyoto Univ. 20 (1980), 749-752.

[AM] M. F. Atiyah and I. G. MacDonald, Introduction to commutative algebra, Addison-Wesley, London, 1969. 
[BCM] J. Brewer, D. Costa and K. McCrimmon, Seminormality and root closure in polynomial rings and algebraic curves, J. Algebra 58 (1979), 217-226.

[D] A. Dixon, A polynomial ring localization: $R\{X\}$, Dissertation, University of MissouriColumbia, 1987.

[G] R. Gilmer, Multiplicative ideal theory, Marcel Dekker, New York, 1972.

[GP] R. Gilmer and T. Parker, Semigroup rings as Prüfer rings, Duke Math. J. 41 (1974), 219-230.

[H] J. Huckaba, Commutative rings, rings with zero divisors, Marcel Dekker, New York, 1988.

[K] I. Kaplansky, Commutative rings, Allyn and Bacon, Boston, 1970.

[La] J. Lambek, Lectures on rings and modules, Blaisdell, Waltham, Mass., 1966.

[ $\mathrm{Lu}_{1}$ ] T. Lucas, Two annihilator conditions: Property $A$ and (a.c.), Comm. Algebra 14 (1986), 557-580.

[ $\mathrm{Lu}_{2}$ ] T. Lucas, Root closure and $R[X]$ (preprint).

[Q] Y. Quentel, Sur la compacité du spectre minimal d'un anneau, Bull. Soc. Math. France 99 (1971), 265-272.

Department of Mathematics, University of North Carolina at Charlotte, Charlotte, North Carolina, 28223 